Rev. Elev. Méd. vét. Pays trop., 1975, 28 (3) : 409-417

\title{
Premiers essais d'embouche de zébus en Haute-Volta
}

\author{
par J. CABARET (*)
}

\begin{abstract}
RESUME
Des essais d'embouche ont été effectués, dans la région de Ouagadougou, sur des zébus de 3 à 5 ans provenant de l'élevage extensif traditionnel, en vue de déterminer la valeur d'une ration à base de graines de coton, pour la production de viande.

Les essais ont duré de 5 à 6 mois, soit en stabulation, soit au pâturage complémenté.

Les premiers essais réalisés en 1972 n'ont donné que de médiocres résultats, mais des gains de poids intéressants (de l'ordre de $550 \mathrm{~g}$ par jour pendant 5 mois) ont été obtenus dans la deuxième série d'essais, sur pâturage complémenté par une ration très économique.
\end{abstract}

\section{Tre PARTIE :}

\section{EXPERIENCE EN 1972}

Le but de l'expérience est de tester la valeur d'une ration à base de graines de coton et de paille de riz, en feedlot permanent en saison sèche; en saison des pluies, les animaux sont entretenus sur pâturage seul. L'embouche dure six mois.

\section{MATERIEL ET METHODE}

\section{I.1. Logement des animaux}

L'expérimentation se déroule à la Station I.R.A.T. de Saria en Haute-Volta.

Deux parcs de stabulation libre sont aménagés sur la base de $10 \mathrm{~m}^{2}$ par animal. Chacun est équipé de mangeoires rateliers (1 poste de $0,50 \mathrm{~m}$ de long par animal), d'auges $(0,50 \mathrm{~m})$ et d'abreuvoirs à niveau constant pourvus d'un compteur d'eau. Le tiers environ de la superficie des parcs, comprenant la rangée des man-

(*) 151 bis, rue Jean Jaurès, 94700 Maisons-Alfort, France. geoires, est abrité du soleil et des intempéries par un toit en tôle. Ils débouchent sur un couloir de régulation et de pesée.

\section{I.2. Animaux}

Les animaux utilisés sont des zébus toutvenant, mâles entiers ou castrés, âgés de 3 à 4 ans et d'un poids moyen de $233 \mathrm{~kg}$, achetés sur les marchés du Nord de la Haute-Volta (Markoye, Dehou).

Dès le regroupement après l'achat, les animaux ont été vaccinés contre la peste bovine et la péripneumonie. Ils sont également traités contre la trypanosomiase et la piroplasmose par le Bérénil et reçoivent un anthelminthique (vadéphène).

Les 46 animaux sont répartis en 3 lots par tirage au sort: deux lots expérimentaux de 18 têtes et un lot témoin de 10 .

\section{I.3. Alimentation}

\section{Aliments utilisés}

L'alimentation est composée :

— d'aliments de lest : 
Paille de riz : 0,34 U.F. et $6 \mathrm{~g}$ de M.A.D. $/ \mathrm{kg}$ M.S.; Foin de Brachiaria: 0,59 U.F. et $7 \mathrm{~g}$ de M.A.D. $/ \mathrm{kg}$ M.S.; Ensilage : 0,22 U.F. et $28 \mathrm{~g}$ de M.A.D. $/ \mathrm{kg}$ M.S.

- de graines de coton de $3^{e}$ choix : 0,88 U.F. et $100 \mathrm{~g}$ de M.A.D. $/ \mathrm{kg}$ M.S.

-- de concentré préparé sur place selon la formule suivante : sorgho grain : $30 \mathrm{~kg}$; maïs : $60 \mathrm{~kg}$; tourteau d'arachide: $9 \mathrm{~kg}$; sel: $1 \mathrm{~kg}$ dont la valeur alimentaire est estimée à : 1 U.F. et $120 \mathrm{~g}$ de M.A.D. $/ \mathrm{kg}$ M.S.

- de minéraux sous forme de pierres à lécher de composition suivante : matières minérales totales : 82 p. 100 minimum dont : phosphore : 8 p. 100 minimum; calcium : 13 p. 100 minimum; chlorures: 38 p. 100 maximum; insoluble chlorhydrique : 3 p. 100 maximum.

\section{Plan d'alimentation}

Le lot témoin (III) est entretenu sur pâturage durant les 6 mois d'embouche.

Lots expérimentaux: $1^{\text {re }}$ phase $(4$ mois de saison sèche)

Lot I : graines de coton; $125 \mathrm{~g}$ de tourteau d'arachide $\mathrm{t} / \mathrm{j}$; Paille de riz.

Lot II : concentré : foin de Brachiaria; ensilage de Pennisetum typhoides.

Les aliments sont distribués à volonté sauf le tourteau et le concentré.

$2^{\mathrm{e}}$ phase

Le lot I : est mis sur pâturage et le lot II reçoit du concentré et de l'herbe à l'auge.

\section{I.4. Observations}

\section{Consommations}

La ration est pesée le matin après la mesure des refus et ajustée à 10 p. 100 supplémentaires de la ration ingérée le jour précédent. La consommation d'eau journalière est relevée tous les matins.

\section{Evolution des poids}

Les animaux sont pesés tous les 15 jours, le matin à jeûn. Les pesées de "référence" mensuelles consistent au passage sur la balance 3 jours consécutifs; la moyeṇne des 3 mesures est retenue.

\section{Examen clinique}

Il est journalier. Des examens coprologiques et hématologiques sont pratiqués sur les animaux dont les gains de poids sont anormaux.

\section{Abattages - Contrôle des carcasses}

A partir de la fin du premier mois et chaque mois ensuite, deux animaux dans chacun des lots expérimentaux et un dans le lot traditionnel sont abattus en vue d'une étude systématique des carcasses. Le critère d'abattage est soit l'appartenance à la classe moyenne, soit le dépassement de la limite des $300 \mathrm{~kg}$.

Les abattages ont eu lieu après un jeûne de $24 \mathrm{~h}$ et les carcasses ont été étudiées.

Le jugement à l'œil, général, est établi sur l'échelle suivante : 0 - nul à 5 - très bon.

La découpe repose essentiellement sur la préparation du pan traité (isolé dès la $6^{\mathrm{e}}$ côte).

\section{RESULTATS}

\section{II.1. Première phase}

(6-3 au 12-7-1972) : 129 jours

- Lot I ( $\mathbf{n}=18)$

a) Evolution des poids (voir tableau $\mathrm{n}^{0} \mathrm{I}$ )

TABLEAU $\mathrm{N}^{\circ} \mathrm{I}$-Evolution des poids et indices de consommation

\begin{tabular}{|c|c|c|c|c|c|c|c|c|c|c|c|c|}
\hline \multirow{3}{*}{ Poids } & $\begin{array}{c}\text { Dates de } \\
\text { pesëes }\end{array}$ & $6-3$ & $20-3$ & $5-4$ & $20-4$ & $9-5$ & $25-5$ & $2-6$ & $13-6$ & $23-6$ & $4-7$ & $12-7$ \\
\hline & Lot I & 228,7 & 224,5 & 226,7 & 232,8 & 229,9 & 240,5 & 235,5 & 234,5 & 242,7 & 246,0 & 245,4 \\
\hline & Lot II & 226,7 & 221,1 & 224,7 & 228,9 & 229,8 & 241,6 & 239,9 & 245,5 & 249,1 & 250,0 & 248,8 \\
\hline moyens & Lot III & 228,9 & 238,5 & 237,8 & 237,2 & 207.4 & 207,3 & 200,3 & 200,3 & 210,8 & $\cdot 228,9$ & 236,8 \\
\hline \multirow{3}{*}{$\begin{array}{l}\text { Indices } \\
\text { de } \\
\text { cons onmation }\end{array}$} & Lot I & & \multicolumn{3}{|c|}{25,3} & \multicolumn{3}{|c|}{44,1} & \multicolumn{2}{|c|}{9,0} & \multicolumn{2}{|c|}{24,1} \\
\hline & Lot II & & \multicolumn{3}{|c|}{92,3} & \multicolumn{2}{|c|}{13,3} & \multicolumn{2}{|c|}{27,4} & \multicolumn{3}{|c|}{30,1} \\
\hline & Lot III & & \multicolumn{10}{|c|}{ non dêterminé } \\
\hline
\end{tabular}


La croissance moyenne est de $130 \mathrm{~g} / \mathrm{t} / \mathrm{j}$.

\section{b) Consommations}

Les animaux s'adaptent difficilement à la vie en feedlot, ce qui retentit sur les consommations au départ. La consommation de graines de coton est faible : $1,39 \mathrm{~kg}$ par animal et par jour.

La ration fournit ainsi :

M.S. : 5,67 kg; U.F. : 2,81; M.A.D. : $203 \mathrm{~g}$

L'indice de consommation est de : 21,1 et la consommation d'eau est de 19,7 l par animal et par jour.

- Lot II $(\mathrm{n}=18)$

a) Evolution des poids (voir tableau $\mathrm{n}^{\circ} \mathrm{I}$ ) Le gain quotidien moyen est de $172 \mathrm{~g} / \mathrm{t} / \mathrm{j}$.

\section{b) Consommations}

M.S. : 8,10 kg; U.F. : 4,72; M.A.D. : 407 g

L'indice de consommation est de 27,5 et la consommation d'eau est de 18,5 l par animal et par jour.

- Lot III $(\mathrm{n}=10)$ a) Evolution des poids (voir tableau $\mathrm{n}^{\circ} \mathrm{I}$ )

Le gain quotidien moyen est de $62 \mathrm{~g}$ par animal et par jour.

- Etude des carcasses (tableau $\mathrm{n}^{\circ}$ II)

\section{II.2. Phase de transition}

Elle consiste au passage au pâturage pour le lot I et au remplacement de l'ensilage et du foin par de l'herbe pour le lot II.

\section{II.3. Phase terminale}

(13-7 au 6-9-1972) : 56 jours

- Lot $\mathrm{I}(\mathrm{n}=12)$

a) Evolution des poids (voir tableau $\mathrm{n}^{\mathrm{a}}$ III)

Le gain quotidien moyen est de $706 \mathrm{~g}$ et l'indice de consommation est de 4,2.

\section{b) Consommations}

Elles sont évaluées à : M.S. : $7,5 \mathrm{~kg}$; U.F. : 3,0; M.A.D. : 225 g.

- Lot II ( $\mathrm{n}=12)$

a) Evolution des poids (voir tableau $\mathrm{n}^{\circ}$ III)

TABLEAU N ${ }^{0}$ II

\begin{tabular}{|l|c|c|c|c|c|c|c|c|c|c|}
\hline \multicolumn{1}{|c|}{ L o t s } & Témoins & \multicolumn{3}{|c|}{ I } & \multicolumn{3}{|c|}{ II } & \multicolumn{3}{c|}{ III } \\
\hline $\begin{array}{l}\text { Nombre animaux } \\
\text { Mesures }\end{array}$ & 2 & 2 & 2 & 2 & 2 & 2 & 2 & 1 & 1 & I \\
\hline $\begin{array}{l}\text { Poids avant } \\
\text { jeûne }\end{array}$ & 233,0 & 232,5 & 236,5 & 240,5 & 238,5 & 236,5 & 269,5 & 231,0 & 205,0 & 223,0 \\
\hline $\begin{array}{l}\text { p.100 perte } \\
\text { au jeûne }\end{array}$ & 9,86 & 5,71 & 4,30 & 4,09 & 5,94 & 6,47 & 4,89 & 4,33 & 4,63 & 13,2 \\
\hline $\begin{array}{l}\text { Poids } \\
\text { carcagse }\end{array}$ & 94,0 & 102,4 & 112,5 & 109,1 & 117,5 & 122,6 & 133,4 & 88,7 & 94,2 & 99,3 \\
\hline $\begin{array}{l}\text { Rendement } \\
\text { vrai en p.100 }\end{array}$ & 53,1 & 53,9 & 56,1 & 54,8 & 58,7 & 59,2 & 56,5 & 48,0 & 53,4 & 56,7 \\
\hline $\begin{array}{l}\text { Poids du 5e } \\
\text { quartier en } \\
\text { p. 100 }\end{array}$ & 24,8 & 25,3 & 27,4 & 25,1 & 25,8 & 27,8 & 28,8 & 25,6 & 27,4 & 29,7 \\
\hline $\begin{array}{l}\text { Gras de rognon } \\
\text { en p. 100 }\end{array}$ & nêant & 0,41 & 0,32 & 0,35 & 0,27 & 0,37 & 0,69 & 0,10 & 0,10 & 0,17 \\
\hline
\end{tabular}

TABLEAU $\mathrm{N}^{\circ}$ III-Evolution des poids.

\begin{tabular}{|c|c|c|c|c|c|c|c|c|}
\hline \multicolumn{2}{|c|}{ Dates de pesêes } & $13-7$ & $25-7$ & $3-8$ & $16-8$ & $23-8$ & $30-8$ & $6-9$ \\
\hline \multirow{3}{*}{ 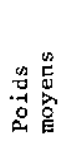 } & Lot I & 245,4 & 249,0 & 255,7 & $26 B, 0$ & 269,3 & $275, B$ & 284,8 \\
\hline & Lot II & 248,8 & 263,7 & 270,1 & 273,6 & 276,9 & 280,2 & 278,7 \\
\hline & Lot III & 236,8 & 250,8 & 256,1 & 270,6 & 273,3 & 279,7 & 283,3 \\
\hline
\end{tabular}


Le gain quotidien moyen est de $533 \mathrm{~g}$.

b) Consommations

M.S. : 9,6 kg; U.F. : 6,1; M.A.D. : 732 g

L'indice de consommation moyen est de 11,4 et la consommation d'eau est de 14,75 par animal et par jour.

- Lot III ( $\mathrm{n}=7$ )

a) Evolution des poids (voir tableau $\mathrm{n}^{\circ}$ III)

Le gain quotidien moyen est de $812 \mathrm{~g}$ par jour.

- Etude des carcasses (tableau $\mathrm{n}^{\circ}$ IV)

\section{ANALYSE}

\section{Evolution des poids}

Les gains de poids moyens ne sont pas significativement (au seuil de 5 p. 100) différents pour les lots I, II, III à la fin de la première phase. Les variances sont très différentes (lots II et III) à la fin de ces quatre mois d'embouche. Par contre les gains de poids sont supérieurs dans les lots en feedlot, par rapport à ceux du lot traditionnel au 25 mai et au 13 juin. L'embouche intensive n'amène donc pas des croîts supérieurs, mais provoque la dissociation des lots en deux groupes: animaux dont la croissance est bonne, animaux dont la croissance est faible ou négative.

A la fin des six mois d'embouche, les gains de poids dans chacun des lots sont presque identiques. Durant la saison des pluies, la croissance "compensatrice " est très importante pour le lot traditionnel.

\section{Evolution des carcasses}

Aucune différence significative n'a pu être mise en évidence entre les lots. Les qualités organoleptiques de la viande sont supérieures dans les lots soumis à l'embouche.

Une corrélation hautement significative a été mise en évidence entre certaines mesures (longueur de carcasse, épaisseur de la cuisse) et le poids de carcasse.

\section{Consommations}

Un laps de temps important est nécessaire aux animaux en feedlot pour s'habituer aux aliments (de 15 jours à 3 semaines) et la consommation de graines de coton est beaucoup plus faible que prévu (moins de $1,5 \mathrm{~kg}$ par animal et par jour). La consommation d'eau n'est pas très élevée : moins de 201 en sajson sèche.

Les diverses consommations sont souvent influencées par le milieu physique (température).

\section{ETAT SANITAIRE}

Il est satisfaisant au cours de l'expérience. Deux animaux ont présenté un syndrome non déterminé (symptômes pulmonaires et locomoteurs), lequel a rétrocédé très rapidement avec un traitement symptomatique. Les lésions sur les carcasses ont été très limitées.

\section{CONCLUSION}

Cette première expérimentation permet de dégager les conclusions suivantes:

1. Le zébu tout venant réagit mal à une embouche intensive. Il semble que l'on puisse

TABLEAU $\mathrm{N}^{\circ} \mathrm{IV}$

\begin{tabular}{|c|c|c|c|c|c|c|}
\hline \multirow[b]{3}{*}{ Poids avant jeûne } & \multicolumn{2}{|c|}{ 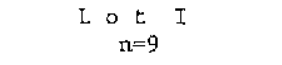 } & \multicolumn{2}{|c|}{$L \circ \underset{n=g}{t} I I$} & \multicolumn{2}{|c|}{$L \circ \underset{n=6}{t}$ III } \\
\hline & Moyenne & $\mathrm{S}$ & Moyenne & $\mathrm{S}$ & Moyenne & $S$ \\
\hline & 276 & 27 & 270 & 45 & 285 & 25 \\
\hline p. 100 perte au jeûne & 7,0 & 1,7 & 5,6 & 1,2 & 6,8 & 1,7 \\
\hline Poids des carcasses & 127,0 & 12,4 & 129,3 & 29,2 & 136,2 & 18,2 \\
\hline Rendement vrai & 56,1 & 2,4 & 57,1 & 3,2 & 56,7 & 4,1 \\
\hline Poids se quartier p.loo & 24,9 & 3,4 & 25,5 & 1,0 & 25,5 & 2,6 \\
\hline
\end{tabular}

$S=E c a r t$ type. 
incriminer l'effet de la claustration comme cause principale; il est très hétérogène et une sélection des animaux les plus aptes s'impose; l'appétence des graines de coton est faible.

Une deuxième expérience était donc nécessaire pour déterminer si la ration à base de graines de coton, en utilisant les principes suivants :

- sélection des animaux;

- diminution du temps de claustration, permet des gains de poids convenables.

\section{2e PARTIE : EXPERIENCE EN 1973}

Le but de l'expérience est de tester la valeur d'une ration à base de pâturage naturel le jour et de graines de coton en feedlot la nuit.

\section{MATERIEL ET METHODE}

Les conditions matérielles, telles que le logement des animaux et les instruments de mesure utilisés sont identiques à celles de 1972. Les abattages sont réalisés en début et fin de saison sèche, en fin d'expérience.

\section{I.1. Les animanx}

Les animaux sont achetés dans les mêmes conditions. Ce sont des mâles entiers ou castrés, âgés d'environ 3 ans et d'un poids moyen de $221 \mathrm{~kg}$. Les 54 animaux sont répartis en 3 lots par tirage au sort : deux lots expérimentaux de 22 têtes et un lot témoin de 10 têtes. Dès le regroupement à Saria, les animaux ont subi les mêmes traitements prophylactiques que l'année précédente.

\section{I.2. Alimentation}

Les aliments utilisés sont de même nature que dans l'expérience précédente mais leur valeur alimentaire est légèrement différente. La paille de riz est de moins bonne qualité alors que le foin de Brachiaria et l'ensilage et les graines de coton ont une valeur supérieure.

Paille de riz : 0,31 U.F. et 6 g M.A.D. $/ \mathrm{kg}$ M.S.; Foin de Brachiaria: 0,63 U.F. et $10 \mathrm{~g}$ M.A.D. $/ \mathrm{kg}$ M.S.; Ensilage : 0,35 U.F. et $47 \mathrm{~g}$ M.A.D./kg M.S.; Graines de coton : 0,92 U.F. et $109 \mathrm{~g}$ M.A.D. $/ \mathrm{kg}$ M.S.
La formule du concentré a été modifiée. La concentration en tourteau d'arachide a été portée à 20 p. 100 la première semaine et ramenée ensuite à 15 p. 100 et le sorgho a été, seul, utilisé, en tant que céréale.

Sorgho grain : $84 \mathrm{~kg}$; Tourteau d'arachide : $15 \mathrm{~kg}$; Sel : $1 \mathrm{~kg}$; U.F. : 1,10/kg M.S.; M.A.D. : $165 \mathrm{~g} / \mathrm{kg}$ M.S.

Le composé minéral est identique à celui de l'expérience 1972.

\section{Plan d'alimentation}

- Lot témoin (III)

Il vit sur pâturage et dispose de pierres à lécher. Il sert de terme de comparaison.

\section{- Lots expérimentaux}

Lot I: Durant les 3 premiers mois (saison sèche) il est au pâturage le jour, et la nuit en feedlot (graines de coton, $250 \mathrm{~g} / \mathrm{t} / \mathrm{j}$ de tourteau, sel et cuivre sous forme de sulfate à raison de 10 p.p.m. de la matière sèche).

En début de saison des pluies (fin juin, début juillet) il est scindé en deux sous-groupes I' et I". Le premier est entretenu sur pâturage seul. Le second est inclus dans le système suivant: pâturage le jour, graines de coton salées la nuit, en feedlot.

Lot II : Durant les trois premiers mois, il reste en feedlot permanent (Paille de riz, ensilage, concentré).

En début de saison des pluies, il est scindé en deux groupes II' et II'. Le premier est entretenu sur pâturage seul comme le lot I'. Le second pâture le jour et reçoit la nuit des graines de coton salées en feedlot.

Pour des raisons de commodité, les lots I", II", sont regroupés dans le même feedlot en saison humide.

\section{RESULTATS}

\section{I.1. Première phase}

(28-3 au 28-6-1973): 93 jours

- Lot I (20 animaux)

a) Evolution des poids (voir tableau $n^{\circ}$ V) 
TABLEAU $\mathrm{N}^{\circ} \mathrm{V}$ - Evolution des poids

\begin{tabular}{|c|c|c|c|c|c|c|c|c|}
\hline $\begin{array}{c}\text { Dates de } \\
\text { pesées }\end{array}$ & $28-3$ & $10-4$ & $26-4$ & $6-5$ & $20-5$ & $4-6$ & $17-6$ & $28-6$ \\
\hline Lot I & 206,2 & 207,2 & 215,2 & 219,0 & 223,7 & 230,6 & 238,8 & 248,7 \\
\hline Lot II & 214,5 & 207,0 & 219,9 & 225,3 & 231,5 & 236,2 & 247,6 & 250,4 \\
\hline Lot III & 209,4 & 215,2 & 201,8 & 196,1 & 188,6 & 198,3 & 208,4 & 215,8 \\
\hline
\end{tabular}

Le gain quotidien moyen est de $456 \mathrm{~g}$.

\section{b) Consommations}

Les animaux s'adaptent rapidement aux graines de coton. La consommation moyenne est de $2,04 \mathrm{~kg} / \mathrm{t} / \mathrm{j}$ et la quantité de fourrage utilisée peut être évaluée à $5 \mathrm{~kg} / \mathrm{j}$. de :

Le bilan alimentaire approximatif est ainsi

M.S. : 7,2 kg; U.F. : 3,6; M.A.D. : 346 g; Indice de consommation : 7,7 .

\section{- Lot II (16 animaux)}

a) Evolution des poids (voir tableau $\Pi^{\circ} \mathrm{V}$ )

Le gain quotidien moyen est de $386 \mathrm{~g}$.

b) Consommations

Les consommations varient au cours de la période, autour des valeurs moyennes suivantes :

Paille de riz : 2,9 kg; Ensilage : 7,0 kg; Concentré : $2,4 \mathrm{~kg}$.

Ce qui conduit au bilan alimentaire suivant:

M.S. : 6,6 kg; U.F. : 4,2; M.A.D. : 458 g; Indice de consommation : 10,8 .

- Lot III (voir tableau $\mathrm{n}^{\circ} \mathrm{V}$ )

Evolution des poids

Le gain quotidien moyen est de $69 \mathrm{~g}$.

- Etude des carcasses (tableau no VI)
TABLEAU N ${ }^{\circ} \mathrm{VI}$

\begin{tabular}{|l|r|r|r|r|}
\hline & $\begin{array}{c}\text { Témoins } \\
n=3\end{array}$ & $\begin{array}{c}\text { Lot I } \\
\mathrm{n}=4\end{array}$ & $\begin{array}{c}\text { Lot II } \\
\mathrm{n}=4\end{array}$ & $\begin{array}{c}\text { Lot III } \\
\mathrm{n}=3\end{array}$ \\
\cline { 2 - 5 } & 209,7 & 250,7 & 262,5 & 224,0 \\
\hline $\begin{array}{l}\text { Pourcent age perte } \\
\text { au jê̂ne }\end{array}$ & 4,26 & 4,08 & 4,65 & 7,71 \\
\hline $\begin{array}{l}\text { Poids carcasse } \\
\text { Poids du 5e }\end{array}$ & 79,3 & 118,0 & 122,5 & 95,0 \\
\hline $\begin{array}{l}\text { Rendement vrai } \\
\text { en p. } 100\end{array}$ & 26,4 & 28,5 & 27,3 & 31,1 \\
\hline
\end{tabular}

\section{II.2. Deuxième phase}

(29-6 au 29-8-1973): 62 jours

- Lots sur pâturage seul

a) Evolution des poids (tableau $\mathrm{n}^{\circ}$ VII)

Les gains journaliers sont: I' : 472 g; II' : $581 \mathrm{~g}$; III : $859 \mathrm{~g}$.

Les animaux éliminés en cours d'expérience ( $1^{\text {er }}$ et $3^{\mathrm{e}}$ mois), eux aussi sur pâturage sont notés (I) et (II) suivant leur ancienne appartenance à un lot. Leurs gains de poids quotidiens moyens sont respectivement de $810 \mathrm{~g}$ et $890 \mathrm{~g}$.

Consommations estimées : U.F. : 5,9; M.A.D. : 262 g.

- Lots au pâturage complémenté.

a) Evolution du poids (tableau $\mathrm{n}^{\circ}$ VII)

Les gains journaliers sont : I" : $673 \mathrm{~g}$; II" : $534 \mathrm{~g}$.

TABLEAU N NII

\begin{tabular}{|l|l|l|l|l|l|l|l|}
\hline Dates des pesées & & $29-6$ & $11-7$ & $25-7$ & $6-8$ & $19-8$ & $29-8$ \\
\hline \multirow{5}{*}{ Poids moyens } & Lot I' & 253,4 & 248,3 & 260,8 & 264,6 & 278,0 & 282,7 \\
\cline { 2 - 8 } & Lot II' & 260,2 & 254,7 & 269,5 & 272,5 & 289,5 & 296,2 \\
\hline & Lot III & 215,9 & 228,6 & 237,6 & 250,4 & 263,1 & 269,1 \\
\cline { 2 - 8 } & Lot I' & 257,6 & 264,7 & 274,0 & 279,8 & 292,4 & 299,3 \\
\hline & Lot II" & 256,5 & 256,2 & 268,0 & 270,9 & 284,7 & 289,6 \\
\hline
\end{tabular}




\section{b) Consommations}

La consommation de graines de coton est en moyenne de $1,5 \mathrm{~kg}$ par animal et par jour.

Compte tenu de la consommation estimée au pâturage, le bilan s'établit comme suit :

M.S. : 6,0 kg; U.F.: 6,4; M.A.D. : 366; Indice de consommation : 9,66 (lot I'); 11,98 (Lot II").

- Etude des carcasses
- La supplémentation du pâturage par des graines de coton présente un intérêt certain pour les animaux qui en ont déjà reçu;

- Ce n'est pas le cas pour les animaux dont la ration en première phase consistait en concentré, ensilage et paille de riz.

Enfin, les animaux éliminés, mis sur pâturage naturel présentent une évolution pondérale semblable à celle du lot traditionnel.

TABLEAU $N^{\circ}$ VIII

\begin{tabular}{|l|c|c|c|c|c|}
\hline & $\begin{array}{c}\text { Lot I' } \\
\mathrm{n}=2\end{array}$ & $\begin{array}{c}\text { Lot II' } \\
\mathrm{n}=2\end{array}$ & $\begin{array}{c}\text { Lot III } \\
\mathrm{n}=2\end{array}$ & $\begin{array}{c}\text { Lot I" } \\
\mathrm{n}=2\end{array}$ & $\begin{array}{c}\text { Lot II" } \\
\mathrm{n}=2\end{array}$ \\
\cline { 2 - 6 } Poids avant jeûne & 282,5 & 305,0 & 270,5 & 307,5 & 293,0 \\
\hline Pourcentage perte au jeûne & 6,01 & 6,33 & 6,30 & 4,28 & 7,2 \\
\hline Poids de carcasse & 132,2 & 146,4 & 125,4 & 249,3 & 145,3 \\
\hline $\begin{array}{l}\text { Poids du 5e quartier en } \\
\text { pourcentage }\end{array}$ & 28,8 & 27,8 & 28,3 & 25,9 & 27,7 \\
\hline $\begin{array}{l}\text { Rendement vrai en } \\
\text { pourcentage }\end{array}$ & 57,6 & 59,1 & 56,6 & 60,3 & 56,5 \\
\hline
\end{tabular}

\section{ANALYSE}

\section{III.1. Evolution des poids}

Les analyses statistiques portent sur les poids moyens des animaux, après une élimination qui est de l'ordre de 10 p. 100 pour le lot I et de 27 p. 100 pour le lot II. Il faut signaler que ce pourcentage est un minimum pour le lot II; si l'on avait retiré tous les animaux de toute évidence inaptes ( 9 sur 22 ), la structure initiale des lots aurait été perturbée et aurait empêché toute analyse statistique sérieuse.

Les tests non paramétriques sur les poids au 18 juin, aussi bien que l'analyse de covariance (29 juin), mettent en évidence les résultats suivants :

- Il existe une différence hautement significative entre les gains de poids des animaux en feedlot et ceux du lot traditionnel durant les 3 mois d'embouche en saison sèche;

- Il n'y a pas de différence entre les gains réalisés par les deux groupes en feedlot.

Au cours de la deuxième phase, en saison des pluies, les résultats (par analyse de covariance) sont, quant aux gains de poids :

\section{III.2. Evolution des carcasses}

Les animaux soumis à l'exploitation en feedlot présentent des carcasses et des bosses plus importantes que celles du lot traditionnel dès la fin de la première phase.

On arrive aux mêmes conclusions en fin de deuxième phase. Les effectifs, lors des abattages, sont faibles et ne permettent pas une pleine exploitation des méthodes statistiques.

On remarquera que les augmentations de poids vifs se répercutent intensément sur celles des carcasses, par exemple pour le lot I" : $65 \mathrm{~kg}$ en plus de carcasse, $85 \mathrm{~kg}$ en plus de poids vif pour 5 mois.

\section{III.3. Consommations}

Les animaux en feedlot permanent (lot II) présentent un temps d'adaptation assez long aux aliments; la consommation de graines de coton s'établit assez vite dans le lot soumis à une semi-claustration; la consommation de pierres à lécher reste en dessous de $15 \mathrm{~g} / \mathrm{t} / \mathrm{j}$.

L'influence des facteurs climatiques sur les consommations a été de nouveau mise en évidence. 


\section{Etat sanitaire}

Les problèmes de santé ont été les plus importants aux alentours du début juin. Le syndrome, d'étiologie inconnue, ressemblant à la fièvre des 3 jours, particularisé par des symptômes pulmonaires et locomoteurs, a touché environ 10 p. 100 de l'effectif, de façon sporadique. Le « syndrome début de saison des pluies" est aisément curable. Le traitement se justifie par la gravité de la maladie, qui peut évoluer vers la mort.

Au cours de la saison des pluies, l'accident majeur est dû aux morsures de serpents qui n'évoluent pas favorablement en général.

La valeur des carcasses est essentiellement diminuée par des lésions de cysticercose.

\section{CONCLUSION}

Cette deuxième expérience d'embouche a pour objectif essentiel de montrer la valeur d'une ration à base de graines de coton et pâturage en groupe semi-contraint. En effet, l'expérience réalisée en 1972 montrait que l'influence de la claustration était très importante et par cela même masquait la valeur réelle de ce type de ration. L'autre facteur mis en évidence était la variation individuelle; des critères de sélection, mis en évidence, demandaient à être vérifiés.

Cette expérimentation permet de dégager les conclusions générales suivantes :

1. La claustration est un élément extrêmement défavorable pour ce type d'animaux. Il faut la réduire au maximum.
2. La sélection s'impose. Les animaux les plus aptes à l'embouche sont caractérisés par : un poil court et rude au toucher; une robe plutôt rouge (éventuellement noire) que blanche; un état sexuel de mâle entier; un poids d'environ $250 \mathrm{~kg}$.

3. Dans ces conditions une ration à base de graines de coton seule, avec pâturage, permet l'obtention de gains de poids très corrects $(550 \mathrm{~g} / \mathrm{t} / \mathrm{j}$ pendant 5 mois $)$.

4. Les carcasses produites sont plus lourdes, plus grasses et donc plus sapides.

La rentabilité de l'opération en milieu paysan est évidente. L'obstacle principal à sa diffusion sera lié à la proportion des animaux inaptes à ce type d'embouche qui provoquera des échecs partiels au niveau de certaines petites exploitations.

La rentabilité de ce genre d'embouche en feedlot industriel dépend essentiellement des charges des structures, et à l'intérieur de cellesci, des dépenses dues aux constructions (étables, hangars de stockage). On n'obtient des taux de rentabilité normaux que dans la mesure où au moins un tiers des constructions emprunte les matériaux et le mode de construction locaux, ce qui est tout à fait réalisable.

Sur le plan technique, des expérimentations complémentaires seraient souhaitables. Elles devraient porter sur l'utilisation des minéraux, et répondre aux questions :

- la supplémentation minérale pour une embouche de 5 mois est-elle rentable?

- quel est le facteur limitant parmi les oligoéléments? sa détermination résoudrait le problème à moindres frais.

\section{SUMMARY}

\section{First trials of zebu cattle fattening in Upper Volta}

Fattening trials were carried out in the Ouagadougou area, on 3 to 5 years old zebu cattle reared under traditional extensive management, with a view to determining the value of a cotton seed based ration for beef production.

The trials were conducted over a 5 to 6 month period, on animals either in feedlots or on pasture with supplementation.

The first 1972 trials gave poor results but significant liveweight gains (about $550 \mathrm{~g}$ by day over a 5 month period) were obtained in the second trial series, on pasture supplemented with a very cheap ration. 


\section{RESUMEN}

Primeros ensayos de engorde de cebues en Alta Volta

Se efectuaron ensayos de engorde, en la región de Uagadugu, en cebues de 3 a 5 años de edad proviniendo de la ganaderia extensiva tradicional, para determinar el valor de una ración con granos de algodón para la producción de carne.

Los ensayos duraron de 5 a 6 meses, bien en estabulación, bien al pasto con aditivos.

Los primeros ensayos, realizados en 1972, no daron más que resultados medianos, pero durante la segunda serie de ensayos se obtuvieron aumentos de peso interesantes (de unos $550 \mathrm{~g}$ por dia durante 5 meses) al pasto con aditivos económicos. 